\title{
Cerebral Cortex Segmentation with Adaptive Fuzzy Spatial Modeling in 3.0T IR - FSPGR MR Images
}

\author{
Syoji KOBASHI*1 - Mieko MATSUI*2 • Noriko INOUE*3 • \\ Katsuya KONDO*1 • Tohru SAWADA*4 • Yutaka HATA*1
}

\begin{abstract}
Measurement of cortical thickness using human brain magnetic resonance (MR) imaging can assist physicians in quantifying cerebral atrophy. Most of the conventional measurement methods assign the same class to all pixels with a similar MR signal independent of their locations, and are therefore unsuitable for MR images that have strong intensity nonuniformity (INU) artifact. We propose an automated method that locally segments the cerebral cortex using an adapted fuzzy spatial model representing the transit of MR signals from the cerebral cortex to the white matter. This method assigns fuzzy degrees belonging to brain tissues using the adaptive fuzzy spatial model for local intensity transition from the cerebral cortex to inside the cerebrum. We also introduce an evaluation method of cortex segmentation algorithms that consists of reproducibility, quantitative, and qualitative tests; we use this method to evaluate and discuss the proposed segmentation method in comparison with the conventional method.
\end{abstract}

Keywords : Cortex Segmentation, Magnetic Resonance Imaging, Medical Imaging, Image Segmentation

\section{Introduction}

The human brain consists of two tissues, which are gray matter (GM) and white matter (WM); the GM on the cerebral surface is called the cerebral cortex. Segmenting cerebral cortex on magnetic resonance (MR) images is a long-standing problem that is still under discussion in the field of medical imaging. It is a fundamental task to quantify cerebral atrophy using MR images; for example, in clinical studies based on tissue classification using MR images, Rusinek et al. reported that, "the percentage of the gray matter in the brains of Alzheimer's disease patients was significantly lower than in control subjects" [1].

Many methods used in classifying the brain tissues or segmenting cerebral cortex on MR images are described in previous studies[2]-[7], and fuzzy logicbased methods have also been studied [8] [9]. Most of the conventional methods are classified as intensity-based approaches, in which pixels with similar

*1 Graduate School of Engineering, University of Hyogo

*2 Department of Radiology, Kakogawa Municipal Hospital

*3 Medical Center for Translational Research, Osaka University Hospital

*4 Department of Rehabilitation, Aino Hospital
MR signals are assigned to the same class regardless of their locations; however, MR signal from brain tissue is not homogeneous due to the intensity nonuniformity (INU) artifact, and the boundary between the brain tissues is unclear due to the partial volume effect (PVE). Therefore, the intensity-based method is not suitable for MR images with INU artifact.

INU artifact is caused by inhomogeneity of the magnetic field; the artifact smoothes the contours of tissues and causes a slight variation in MR signal on the MR images. INU artifact is a particularly serious problem in the high-field MR scanners (3 tesla or higher) that enable visualization of the small changes in brain tissues[10]. Some methods correct for INU artifact (e.g.,[11][12]); however, it is still difficult to precisely estimate INU because INU is unstable. Although several methods have been proposed that locally classify the brain tissues [13] [14] and address INU artifact, the classification performance, computation cost, and difficulty in the implementation of these methods are still under discussion.

In the present study, we propose a cerebral cortex extraction method using 3.0 tesla inversion recovery 
fast spoiled GRASS (IR-FSPGR) MR imaging. The algorithm addresses INU artifact by locally adapting a fuzzy spatial model of MR signals that transit the GM and WM. Thus, in contrast to conventional methods, the proposed algorithm assigns fuzzy degrees that are dependent on location to pixels belonging to GM or WM.

The validation of classification methods is very difficult because of the lack of a gold standard. Luo et al. introduced a method that uses a simulated brain database (SBD) [15]; however, it is difficult to emulate a given MRI scanner or subject. In the present study, we introduce a validation method that does not use simulated data. The validation method consists of three tests - reproducibility, quantitative, and qualitative tests - and can be performed by employing multiple image acquisition of the same subject and using a questionnaire test for experts. The present article evaluates the proposed segmentation method based on the introduced validation tests, and compares its performance to the conventional method [5] that was implemented by FMRIB's Software Library(FSL) [16].

\section{Subjects and Materials}

This study recruited two normal healthy subjects: Subject A (female; age, 34 years) and Subject B (male; age, 35 years). Both subjects gave their informed consent according to the guidelines approved by the Ethical Committee of the BF Research Institute Inc., Japan.

MR scanning was performed using a 3.0 tesla SIGNA LX VH/i scanner (GE Medical Systems, Milwaukee, WI, USA), with a circularly polarized head coil. Coronal three-dimensional IR-FSPGR images were acquired using the following imaging parameters: repetition time (TR), $10.7 \mathrm{~ms}$; actual echo time (TE), 1.9 $\mathrm{ms}$; inversion time (TI), $600 \mathrm{~ms}$; field of view (FOV), $220 \times 220 \mathrm{~mm}$; matrix, $256 \times 256$. Each volume dataset consisted of 124 separate volumetric slices with a thickness of $1.5 \mathrm{~mm}$ and no gap; voxel size was $0.86 \times$ $0.86 \times 1.5 \mathrm{~mm}$. Multiplanar reconstructed (MPR) MR images are shown in Fig.1.

The 124 acquired slices were reconstructed into a volume dataset with the dimensions $256 \times 256 \times 124$ voxels. The MR signals for all voxels of all intracranial structures ranged from 0 to 4095 . The acquired

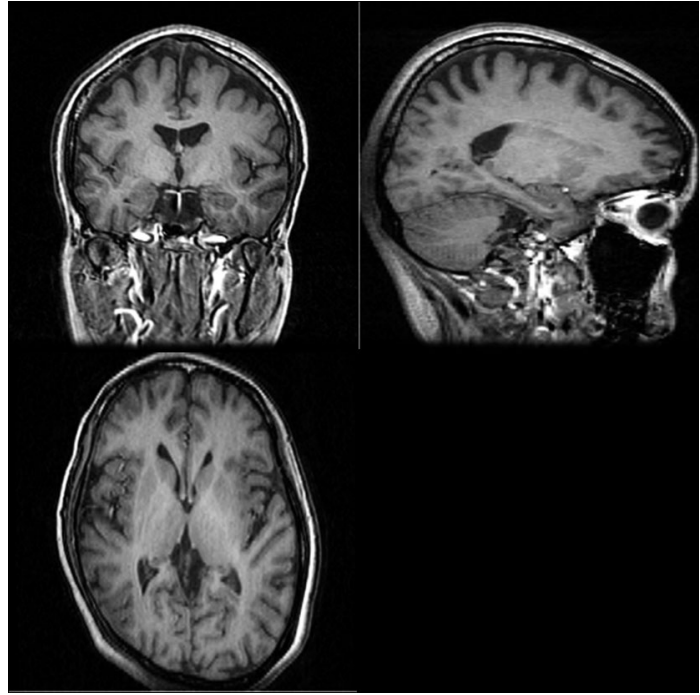

Fig.1 Multiplanar reconstructions of representative MR images. Upper left, coronal; upper right, sagittal; and lower left, axial.

volume data were translated into a Talairach coordinate system and segmented into the left and right cerebral hemispheres, cerebellum, and brain stem using a previously described automated human brain MR image segmentation algorithm[17] [18].

\section{Adaptive Classification with Fuzzy Spatial Modeling}

This article proposes a voxel-based method for segmentation of the cerebral cortex. The principal concept behind the proposed method is illustrated in Fig.2. According to a line that is drawn from the cerebral contour to the inside of the cerebrum, MR signal changes from a low value to a high value. Many lines can be drawn from the cerebral contour to the inside; however, one line can be found in which the
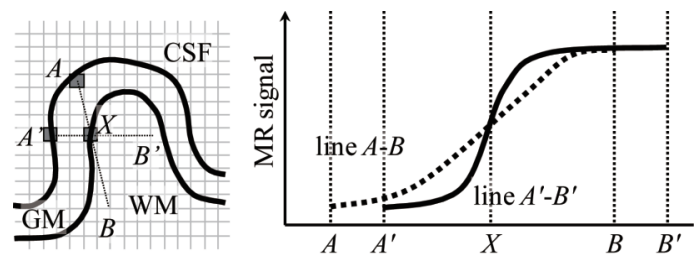

Fig.2 Principal concept of the proposed method. Left, part of a gyrus; right, comparison of MR signal transition between $A-B$ and $A^{\prime}-B^{\prime}$. $\mathrm{X}$ is the point on the boundary between the cerebral cortex and the WM. 
MR signal clearly changes from a lower value to a higher value; for example, assuming the section of gyrus illustrated in Fig.2 and comparing MR signal transitions for $A-B$ and $A^{\prime}-B^{\prime}$. The line segments $A-B$ and $A^{\prime}-B^{\prime}$ are termed searching segments (SSs) in the present study. In the case of $A-B$, the MR signal changes gradually because of PVE; in contrast, for $A^{\prime}-B$ ' the MR signal changes steeply. In this case, the boundary between the cerebral cortex and WM (termed the tissue boundary) is better determined using $A^{\prime}-B^{\prime}$ than $A-B$. Based on this concept, our classification algorithm introduces two fuzzy degrees for an SS: the first degree assigns a degree of belonging to the cerebral cortex for voxels on the SS, while the second degree provides a degree of certainty as to whether it is easy to find the MR signal change at the tissue boundary.

The proposed method first assigns two fuzzy degrees for the cerebral voxels using many SSs drawn from voxels on the cerebral contour. That is, for one voxel, many sets of the two fuzzy degrees are assigned from many SSs. Second, for each voxel, the fuzzy degree belonging to the cerebral cortex with respect to all of the SSs is calculated from the set of the two fuzzy degrees. Finally, according to the calculated fuzzy degree the voxel is classified as cerebral cortex or WM. In summary, the proposed method consists of three steps: (1) fuzzification that assigns fuzzy degrees, (2) fuzzy calculus that calculates the fuzzy degree belonging to cerebral cortex, and (3) defuzzification that classifies the voxels into cerebral cortex and WM. The details of each step are described below.

\subsection{Fuzzification with Adaptive Fuzzy Spatial Modeling}

SSs are drawn from all voxels on the cerebral contour (called starting points) to the inside of the cerebrum in all directions, with length $L$. For example, for a two-dimensional image, the number $n$ of SSs is drawn from a voxel $p$ on the cerebral contour to the inside of the cerebrum, as shown in Fig.3. For each SS, we estimate two types of fuzzy degree: $\mu_{C C}$ and $\mu_{c}$, which are assigned for each voxel on the SS.

$\mu_{C C}$ is a fuzzy degree regarding whether the voxel of interest (VOI) is cerebral cortex with respect to the SS, and is estimated for each voxel on the SS. To estimate this fuzzy degree we construct a model that simulates the ideal MR signal transition on the SS, as

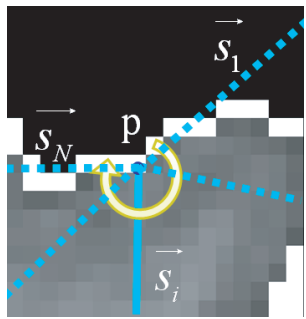

Fig.3 Searching segments(SSs). Blue lines are SSs that are drawn from starting voxel $p$.

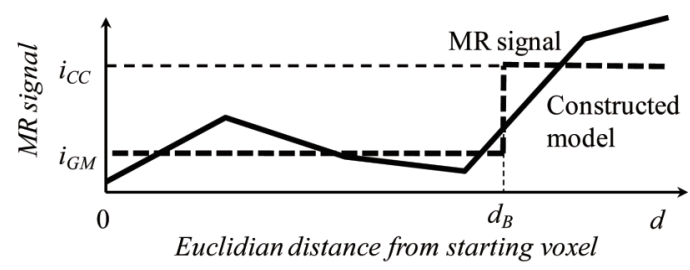

Fig.4 MR signal transition model on an SS. Solid line, MR signal; dotted line, constructed model.

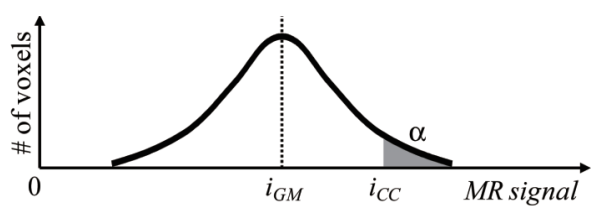

Fig.5 MR signal distribution of cerebral voxels inside the sphere.

illustrated in Fig.4, and formulated by

$$
i(d)=\left\{\begin{array}{ll}
i_{C C} & d<d_{B} \\
i_{W M} & d \geq d_{B}
\end{array},\right.
$$

where $d[\mathrm{~mm}]$ and $d_{B}[\mathrm{~mm}]$ are Euclidian distances from starting voxel $\mathrm{p}$ to the VOI, and to the tissue boundary, respectively; and $i_{C C}$ and $i_{W M}$ are ideal MR signals of cerebral cortex and WM, as estimated below.

We assume a sphere whose center is voxel p and whose radius is $R$. When $R$ is smaller than or equal to the thickness of WM, most of the voxels in the sphere will be the cerebral cortex or CSF voxels. Thus, the MR signal distribution of the cerebral voxels within the sphere will resemble a normal distribution, as shown in Fig.5. We then approximate the obtained MR signal distribution of the cerebral cortex voxels using a normal distribution whose shape is determined by the center MR signal and the variance of distribution 


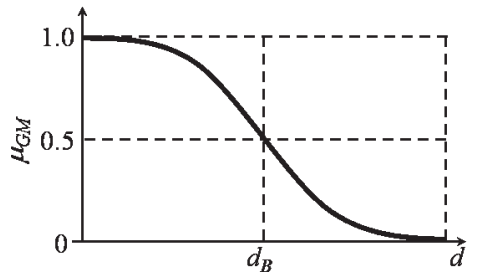

Fig.6 Fuzzy degree belonging to GM.

using the least square method (LSM). MR signals of WM voxels are significantly higher than those of cerebral cortex voxels; thus, we set the MR signal of $\mathrm{WM}, i_{C C}$, at an MR signal whose percentage point on the upper normal distribution is $\alpha$ percent, and the MR signal of cerebral cortex, $i_{G M}$, at the center MR signal of the normal distribution. Using the constructed model, $\mu_{C C}$ is estimated by

$$
\begin{aligned}
\mu_{C C}(d) & =s\left(d_{B}-d\right) \\
& =\frac{1}{1+\exp \left(-d_{B}+d\right)},
\end{aligned}
$$

where $s()$ represents the sigmoid function shown in Fig.6. A VOI close to the cerebral contour has a high $\mu_{C C}$; in contrast, a VOI far from the estimated tissue boundary (within the cerebrum) has a low $\mu_{C C}$.

The constructed model can be stretched or flexed by changing the parameter $d_{B}$, which is the distance from the cerebral contour to the tissue boundary. Thus, the LSM can be used to find the most suitable parameter such that the model constructed by the parameter is most similar to the MR signal transition on the SS.

To evaluate the confidence of use of the SS of interest, a confidence factor is estimated for the SS. Considering the MR signal transition shown in Fig.4, let the sum of the squared errors (SSE) between the MR signal transition and the constructed model be $E_{\text {sum }}$ (Fig.7 (a)) and the SSE between the MR signal transition and $i_{C C}$ be $E_{\max }($ Fig.7 (b)). The definitions of $E_{\text {sum }}$ and $E_{\max }$ are formulated by

$E_{s u m}=\sum_{d=0}^{L}[f(d)-i(d)]^{2}$

and

$E_{\max }=\sum_{d=0}^{L}\left[f(d)-i_{C C}\right]^{2}$,

where $f(d)$ is MR signal for a voxel whose distance is $d$. When $E_{\text {sum }}$ is equal to zero, the MR signal transition

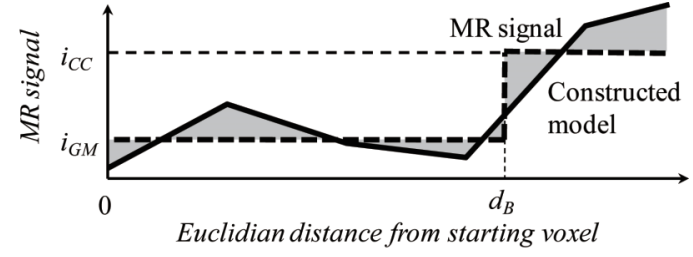

(a) Definition of $E_{\text {sum }}$.

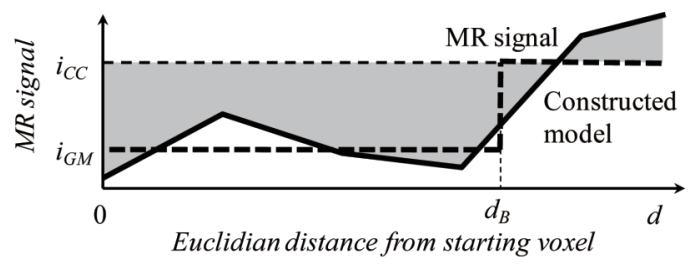

(b) Definition of $E_{\max }$.

Fig.7 Definition of the sum of squared the errors. Solid line, MR signal; dotted line, constructed model.

is identical to the ideal MR signal transition. When $E_{\text {sum }}$ equals or exceeds $E_{\max }$, the MR signal transition does not resemble the ideal MR signal transition. For example, in the case of the two MR signal transitions shown in Fig.2, $E_{\text {sum }}$ of $A-B$ is higher than that of $A^{\prime}-$ B'.

Thus, we define the confidence factor $\mu_{c}$, which measures whether the fuzzy degree $\mu_{C C}$ is estimated with certainty using the SS, as

$\mu_{c}=1.0-\frac{E_{\text {sum }}}{E_{\max }}$,

when $E_{\text {sum }}$ is smaller than or equal to $E_{\max }$; otherwise, $\mu_{c}$ is set to zero. This confidence factor takes lower values when $E_{\text {sum }}$ is similar to $E_{\max }$.

\subsection{Fuzzy Calculus and Defuzzification}

This section introduces a method for calculating the fuzzy degree $\left(\mu_{C C}{ }^{\text {total }}\right)$ belonging to the cerebral cortex, with respect to all SSs. Consider voxel p, which is a voxel of the cerebrum. Let the number of SSs that run through the voxel $\mathrm{p}$ be $N_{s}(\mathrm{p})$, and the two fuzzy degrees that are assigned by the $i$ th $\left(1 \leq i \leq N_{s}(\mathrm{p})\right)$ SS be $\mu_{c}(\mathrm{p}, i)$ and $\mu_{W M}(\mathrm{p}, j) . \mu_{C C}{ }^{\text {total }}$ is calculated by

$\mu_{C C}^{\text {totati }}(\mathrm{p})=\frac{\sum_{i=1}^{N_{s}(\mathrm{p})} \mu_{c}(\mathrm{p}, i) \times \mu_{C C}(\mathrm{p}, i)}{\sum_{i=1}^{N_{s}(\mathrm{p})} \mu_{c}(\mathrm{p}, i)}$, 
where $N_{s}(\mathrm{p}) \neq 0$. When $N_{s}(\mathrm{p})$ is zero, $\mu_{G M}{ }^{\text {total }}$ is set to zero. The fuzzy degrees $\mu_{G M}$ total are calculated by applying Eq. (6) to all voxels in the cerebrum; they take a value between 0 and 1 . A fuzzy degree of 0 means that the voxel does not belong to cerebral cortex perfectly, i.e., the voxel belongs to WM perfectly. A fuzzy degree of 1 means that the voxel belongs to cerebral cortex perfectly, i.e., the voxel does not belong to WM perfectly.

Defuzzification is a process that enables a crisp value to be derived from a fuzzy degree. In this study, the crisp value corresponds to the label of cerebral cortex or WM. Because the fuzzy calculus process gives a fuzzy degree between 0 and 1 , and the fuzzification process assigns a value of 0.5 for a voxel on the estimated tissue boundary, the fuzzy degree $\mu_{G M}{ }^{\text {total }}$ is defuzzified by a simple thresholding with a parameter of 0.5 .

As an example of fuzzy calculus and the defuzzification process, consider the VOI and two SSs $\left(\mathrm{SS}_{\mathrm{A}}\right.$ and $\left.\mathrm{SS}_{\mathrm{B}}\right)$ shown in Fig.8. In this figure, $d_{B}(A)$ and $d_{B}(B)$ (denoted by solid lines) are the estimated boundaries in the fuzzification process. At this time, $d_{B}(B)$ had been misestimated due to PVE. Let the confidence factors for $\mathrm{SS}_{\mathrm{A}}$ and $\mathrm{SS}_{\mathrm{B}}$ be $\mu_{C}(A)$ and $\mu_{C}(B)$, respectively, and the VOI's fuzzy degrees for belonging to the cerebral cortex with respect to $\mathrm{SS}_{\mathrm{A}}$ and $\mathrm{SS}_{\mathrm{B}}$ be $\mu_{C C}(A)$ and $\mu_{C C}(B)$, respectively. In this case, because $\mathrm{SS}_{\mathrm{B}}$ runs along the tissue boundary, $\mu_{C}(B)$ is smaller than $\mu_{C}(A)$ due to PVE. For example, in the case of $\mu_{C}(A)=0.9, \mu_{C}(B)=0.2, \mu_{C C}(A)=0.7$, and $\mu_{C C}(B)=0.3, \mu_{G M}{ }^{\text {total }}$ is calculated as

$$
\begin{aligned}
\mu_{C C}^{\text {total }}(\mathrm{VOI}) & =\frac{0.9 \times 0.7+0.2 \times 0.3}{0.9+0.2} . \\
& =0.63
\end{aligned}
$$

The VOI is correctly classified as WM. If we had classified the VOI using only $\mathrm{SS}_{\mathrm{B}}$, the VOI would have

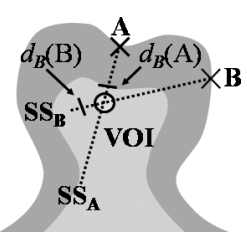

Fig.8 Example of the method used in calculating the resultant fuzzy degree. been classified as cerebral cortex because $\mu_{C C}(\mathrm{~B})$ is higher than 0.5. This example demonstrates that the proposed method classifies the brain tissues using an appropriate SS where the tissue boundary on the MR signal transition is clearly described.

\section{Evaluation and Comparison Methods}

Conventional methods for evaluating tissue classification algorithms are based on phantom data in a computer simulation [15], or based on a slice-by-slice comparison between automatically determined and manually delineated boundaries. However, it is difficult to simulate real MR images using a computer, manual evaluation of the numerous slices using real MR images is a time-consuming approach, and there is variability in intra- and inter-user evaluation performance. These evaluation methods cannot evaluate whether the algorithm can be used for the purpose of deriving a second opinion, or whether it is a stable method suitable for calculating volumes for the purpose of diagnosing the aging of tissue over time.

Thus, we consider that tissue classification algorithms should be evaluated using three kinds of tests: reproducibility, quantitative, and qualitative. A classification methodology that shows good results in all three evaluation tests is stable and valid. Details of the evaluation tests are described below.

\subsection{Reproducibility Test}

This test aims to evaluate variation in volume among the acquisitions: the testing method measures volumes of cerebral cortex and WM using several sets of MR images taken from one subject over a short period. Assume that the brain tissue volumes of normal subjects remain almost constant over a short period, and that the performance of the MR device is steady over this duration. We acquired MR images 10 times (twice per day) within 14 days for Subjects $\mathrm{A}$ and $\mathrm{B}$; we applied the proposed method to these images to validate the reproducibility of the measured volumes of cerebral cortex and WM. If the tissue classification method is steady, the same volume can be obtained from any MR image of the same subject. Thus, the variance in cerebral cortex or WM volumes denotes the performance in terms of reproducibility. 


\subsection{Quantitative Test}

This test evaluates whether the testing method can represent an experienced radiologist in delineating the tissue boundary. This test, which can be classified as a conventional human-based evaluation, compares WM volumes measured by the testing method with those measured by manual delineation. Because it is difficult and time-consuming to manually delineate all boundaries in all slices, we tested eight gyri: anterior central gyrus $(\mathrm{ACG})$, posterior central gyrus (PCG), superior frontal gyrus $(\mathrm{SFG})$, and superior temporal gyrus (STG) in both hemispheres at defined testing locations.

$\mathrm{WM}$ volumes at the SFG and STG are measured on coronal images. Let Section A be a coronal section in which a line connecting the central sulci in both hemispheres crosses the longitudinal fissure of cerebrum (LFC), and let Section B be a coronal section including the most anterior part of the cerebrum. The three coronal sections that divide the space between Sections $\mathrm{A}$ and $\mathrm{B}$ into four capitations are the test coronal images $\left(\mathrm{TCI}_{1}, \mathrm{TCI}_{2}, \mathrm{TCI}_{3}\right)$. The test coronal images are shown in Fig.9 (a).

WM volumes at the ACG and PCG are measured on sagittal images. Let a sagittal section running along the LFC be Section X, and sagittal sections that include the left (or right) end of the cerebrum be Section $\mathrm{Y}$ ( or $\mathrm{Z}$ ). The four sagittal sections that divide

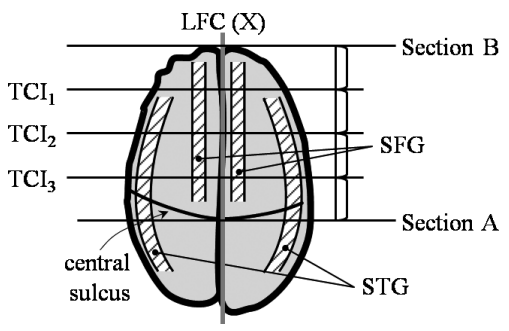

(a) Coronal evaluation images.

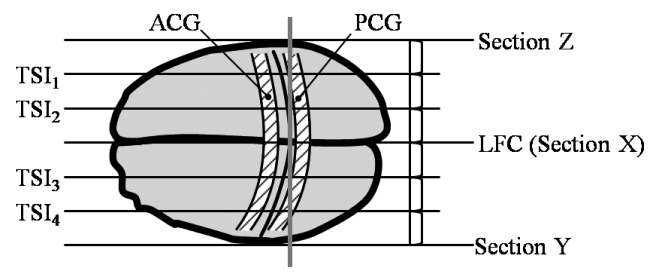

(b) Sagittal evaluation images.

Fig.9 Definition of evaluation sections.

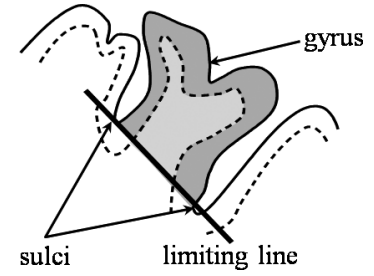

Fig.10 Testing area.

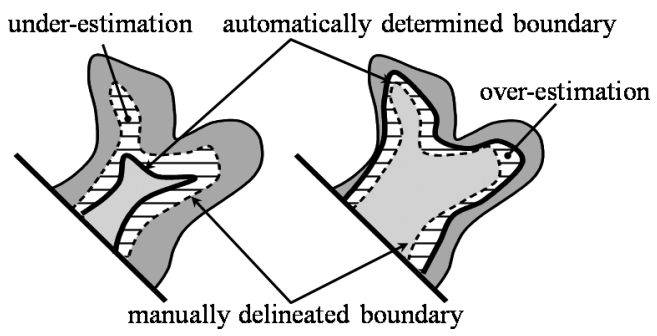

Fig.11 Under- and over- estimation.

the space between Sections $\mathrm{X}$ and $\mathrm{Y}$ or between $\mathrm{X}$ and $\mathrm{Z}$ into three capitations are the test sagittal images $\left(\mathrm{TSI}_{1}, \mathrm{TSI}_{2}, \mathrm{TSI}_{3}, \mathrm{TSI}_{4}\right)$. The test sagittal images are illustrated in Fig.9(b).

The WM volume of a given gyrus on a given testing image is measured within a testing area divided by a line, as shown in Fig.10. The line is placed by an assessor, who first segments the WM in the testing area by manually delineating the boundary. We used the manually segmented area as the ground-truth area before calculating the volumes of the correctly estimated area (TP), the under-estimation area $(\mathrm{FN})$, and the over-estimation area (FP), as shown in Fig.11.

\subsection{Qualitative Test}

This test evaluates whether multiple doctors approve the classification result produced by the testing method. For this purpose, we employed multiple assessors. For each assessor we presented both an enlarged raw MR image at the given gyrus and the automatically determined tissue boundary superimposed on the MR image on a computer display, as shown in Fig.12. The MR images were chosen in the same manner as that described in Section 4.2. According to their knowledge and experience, the assessors are required to evaluate the validity of each image presented in brain anatomy and MR imaging until the next testing image is displayed; the answers are provided as one of five options, as tabulated in Table 1. 


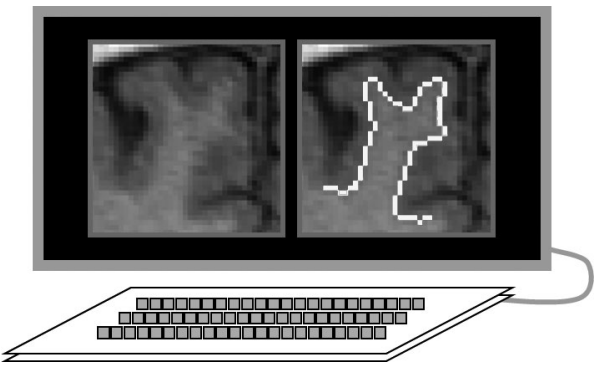

Fig.12 Qualitative test. Left; raw MR image, right; segmented result.

Table 1 Evaluation options

\begin{tabular}{|l|l|}
\hline Evaluation & Meaning \\
\hline \hline A (good) & WM is correctly segmented \\
\hline $\mathrm{B}$ (over) & WM is over-segmented \\
\hline $\mathrm{C}$ (under) & WM is under-segmented \\
\hline $\mathrm{D}$ (mix) & $\begin{array}{l}\text { Over-segmentation and under-segmentation } \\
\text { of WM are seen }\end{array}$ \\
\hline $\mathrm{E}$ (unknown) & $\begin{array}{l}\text { Validation is difficult using only the given } \\
\text { image }\end{array}$ \\
\hline
\end{tabular}

In this evaluation test, the assessors were blinded to any information regarding the subject (such as name, gender, age, and medical history) and to the computation method applied to avoid biased evaluation.

\section{5 . Experimental Results}

\subsection{Reconstructed Sectional Images}

In the present study, we used an SS length $L$ of 15.0 $\mathrm{mm}$, radius of the sphere $R$ of $5.0 \mathrm{~mm}$, and significance level $\alpha$ of 0.02 for all experiments. These parameters were chosen subjectively through our experiments. Fig.13 shows a raw coronal image of Subject A, the fuzzy degree map, and the classification result obtained using the proposed method. In the fuzzy degree map, the fuzzy degree $\mu_{G M}{ }^{\text {total }}$ is presented using a red-yellow-white color scale; the white end of the scale denotes a higher fuzzy degree. The figure shows that the higher fuzzy degrees were assigned to pixels located close to the cerebral contour.

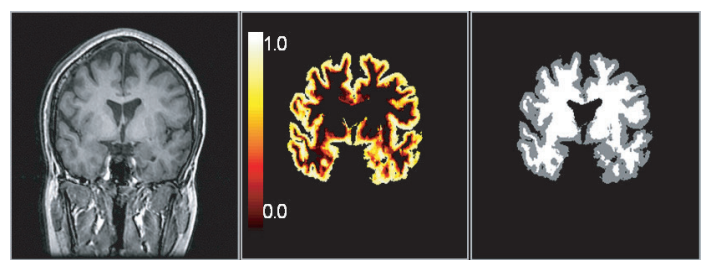

Fig.13 Experimental result. Left, raw image; center, fuzzy degree map; right, classification result.
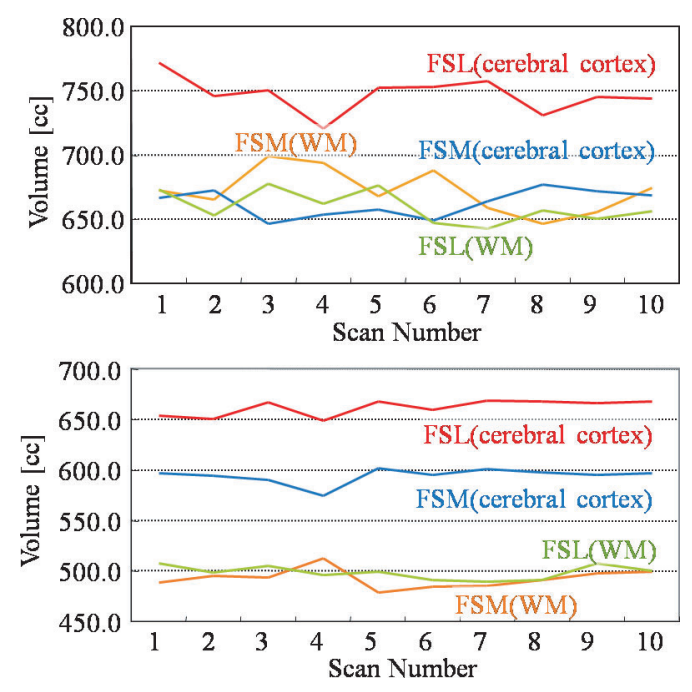

Fig.14 Reproducibility test and comparison. Upper, Subject A; lower, Subject B.

Table 2 Mean and SD of the measured cerebral cortex volume (cc). Values in parentheses denote the ratio of $\mathrm{SD}$ to mean.

\begin{tabular}{|c|c|c|}
\hline Subject & FSM & FSL \\
\hline \hline A & $662.4 \pm 10.9(1.6 \%)$ & $747.0 \pm 14.8(2.0 \%)$ \\
\hline B & $597.3 \pm 3.3(0.6 \%)$ & $663.6 \pm 6.8(1.0 \%)$ \\
\hline
\end{tabular}

\subsection{Reproducibility with Multiple Image Acquisitions}

For each subject, we acquired images 10 times (twice per day) on 5 days over a period of 14 days. Fig.14 shows the variability in cerebral cortex and WM volumes measured using the proposed method (fuzzy spatial modeling (FSM)) and the conventional method (FSL) [16]. The mean and standard deviation (SD) of the cerebral cortex volume is tabulated in Table 2. This table shows that FSM had higher reproducibility in measuring cerebral cortex volumes than FSL.

\subsection{Quantitative Test with Ground Truth Data}

The quantitative test was applied to the MR images of each subject. For each gyrus, an experienced radiologist manually delineated the tissue boundary on the three defined coronal images or four defined sagittal images, and measured the volumes of the TP, FN, and FP areas. Using the measured volumes, we then calculated sensitivity, which we defined as TP/ $(\mathrm{TP}+\mathrm{FN})$, and mean sensitivity for each gyrus.

Fig.15 shows the mean sensitivity for each gyrus 


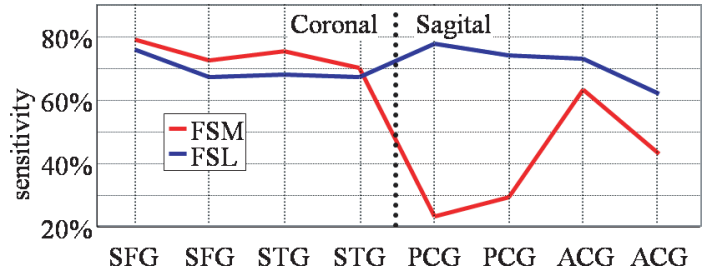

Fig.15 Mean sensitivity of WM segmentation at each gyrus for Subject A.

that was segmented by FSM and FSL. In this figure, SFG and STG were assessed on coronal images, while PCG and ACG were assessed on sagittal images. This figure shows that FSM segmented WM with a higher accuracy than FSL for assessments performed on coronal images; however, on the sagittal images, sensitivity using FSM was lower than that using FSL. The differences in sensitivity between the assessment sections are caused by differences in the spatial resolution of the MR images between the body axis $(1.5 \mathrm{~mm}$ / voxel) and the coronal image ( $0.86 \mathrm{~mm} /$ voxel). Similar results were obtained for Subject B.

\subsection{Qualitative Evaluation with a Blind Questionnaire Test}

To perform this test, we employed seven assessors (five neurosurgeons, one senior physician, one brain MR researcher) who are well versed in brain anatomy and MR images. We constructed test images using results obtained by FSM (40 images) and FSL (80 images). In addition, 40 images selected randomly from the 120 testing images were duplicated to evaluate the reliability of the assessments. The 160 images were then randomly divided into 4 sets. One set consisting of 40 images was continuously displayed for $10 \mathrm{~min}$ (15 sec per image with an interval of $5 \mathrm{sec}$

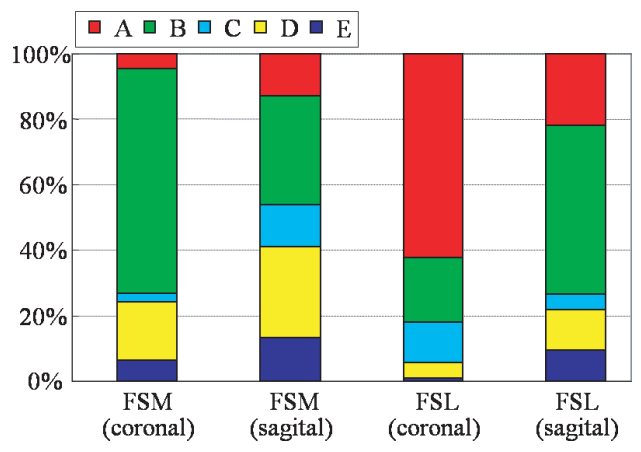

Fig.16 Qualitative evaluation test. between images); the displayed image was automatically changed to the next test image. A 5 min resting period was taken between the evaluation sets.

The results of this test with FSM and FSL are summarized in Fig.16; the results are divided into those assessed on coronal images and those assessed on sagittal images. The figure reveals that the proposed method has a tendency toward the over-segmentation of WM.

\section{Discussions}

Fig.17 (a) shows a comparison of segmentation results using FSM and FSL for a gyrus with a simple shape. In this case, FSL over-segmented the WM area because there was high MR signal due to PVE, while FSM correctly found a boundary similar to that delineated manually. We consider that in this case FSM found the correct boundary by setting SSs in the direction that clearly described the boundary.

Fig.17 (b) shows the results for a gyrus with a complex shape. In this case, FSL found a more appropriate boundary in comparison with FSM. Because the MR signal transition model (Fig.4) used in FSM cannot represent such complex gyri, the boundary estimated by FSM becomes the smoothed curve shown in Fig.17(b). Considerations for future study include the development of a methodology that enables the SS length to change dynamically according to the complexity of the gyri, another model fitting method, and another model.

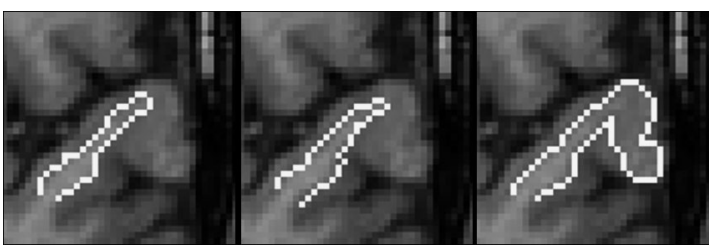

(a) Simple shaped gyrus.

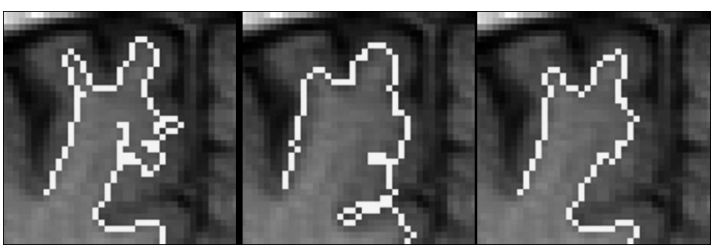

(b) Complex shaped gyrus.

Fig.17 Segmentation comparison with FSL. Left, manual; center, FSM; right, FSL. 


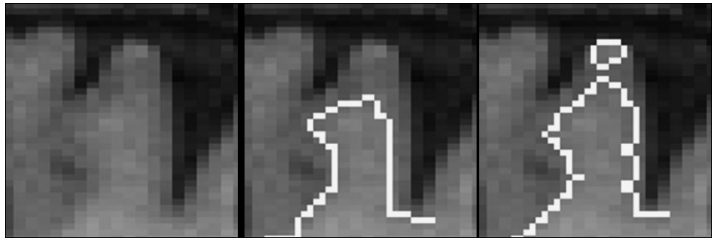

Fig.18 Misclassification with FSL. Left, raw image; Center and right, segmentation results with FSM and FSL, respectively.

Fig. 18 shows a case in which FSL classified voxels on the cerebral contour as WM, even though there is no WM on the cerebral contour. Misclassification such as this occurs with FSL because FSL is an intensity-based method, and the misclassified voxels have high MR signal similar to the MR signal of WM due to INU artifact; however, the appropriate boundary was found using FSM, which is a shape-based method.

In summary, the proposed method enables accurate classification results in the case of gyri with a simple shape and obtains appropriate results that are not limited by INU artifact. The limitation of the method is that in the case of gyri with a complex shape, the estimated boundary is smoothed due to mis-fitting of the MR signal transition model.

\section{Conclusions}

This article proposes a novel method for segmenting cerebral cortex on MR images. The method detected the boundary between the cerebral cortex and WM using a spatial model locally. The spatial model was applied using various locations and with various directions; the method found the appropriate tissue boundary by integrating the results with various spatial models using fuzzy degrees. To validate the proposed method, we introduced three tests: reproducibility, quantitative, and qualitative. The validation tests for the proposed method showed that the method is effective in classifying brain tissues when MR signal varies locally due to INU artifact and PVE effect, among others.

A limitation of the method is that it cannot be applied to GM deep inside the cerebrum. In the future, we will study a fine-measurement method using subvoxels, and introduce an intensity-based approach into the proposed method to develop a new method that combines the advantages of the intensity-based and voxel-based methods. We should also investigate the dependency of analysis parameters on segmentation results, and conduct research to decrease the parameters.

\section{Acknowledgements}

We thank PhD candidates M. Umeda (Dept. of Medical Informatics, Meiji University of Oriental Medicine) and M. Fukunaga (BF Research Institute Inc. and Dept. of Medical Informatics, Meiji University of Oriental Medicine) for their advice on 3T-MRI. We also thank Mr. S. Itoi (BF Research Institute Inc.) for his assistance in MR data acquisition. This work was supported in part by a Grant-in-Aid for the Encouragement of Young Scientists from Ministry of Education, Culture, Sports, Science and Technology (MEXT), JAPAN, a grant from the Ishikawa Hospital, and by the Berkeley Initiative in Soft Computing (BISC) Program of UC Berkeley, USA.

\section{References}

[1] H. Rusinek, M. J. de Leon, A. E. George, L. A. Stylopoulos, R. Chandra, G. Smith, T. Rand, M. Mourino and H. Kowalski, "Alzheimer Disease: Measuring Loss of Cerebral Gray Matter with MR Imaging," Radiology, Vol.178, No.1, pp.109-114, 1991.

[ 2 ] S. Ruan, C. Jaggi, J. Xue, J. Fadili and D. Bloyet, "Brain Tissue Classification of Magnetic Resonance Images Using Partial Volume Modeling," IEEE Trans. on Medical Imaging, Vol.19, No.2, pp.1179-1187, 2000.

[ 3 ] D. H. Laidlaw, K. W. Fleischer and A. H. Barr, "Partial-Volume Bayesian Classification of Material Mixtures in MR Volume Data Using Voxel Histograms," IEEE Trans. on Medical Imaging, Vol.17, No.1, pp.7486, 1998.

[ 4 ] K. Van Leemput, F. Maes, D. Vandermeulen and P. Suetens, "Automated Model- Based Tissue Classification of MR Images of the Brain," IEEE Trans. on Medical Imaging, Vol.18, No.10, pp.897-908, 1999.

[ 5 ] Y. Zhang, M. Brady and S. Smith, "Segmentation of Brain MR Images through a Hidden Markov Random Field and the Expectation-Maximization Algorithm," IEEE Trans. on Medical Imaging, Vol.20, No.1, pp.4557, 2001.

[6] J-F. Mangin, O. Coulon and V. Frouin, "Robust Brain Segmentation Using Histogram Scale- Space Analysis and Mathematical Morphology," Lecture Notes in Comp. Sci., MICCAI, Vol.1496, pp.1230-1241, 1998.

[ 7 ] V. Grau, A. U. Mewes, M. Alcaniz, R. Kikinis and S. K. Warfield, "Improved Watershed Transform for Medical Image Segmentation Using Prior Information," IEEE Trans. on Medical Imaging, Vol.23, No.4, pp 447458, 2004. 
[ 8 ] A. W. Liew and H. Yan, "An Adaptive Spatial Fuzzy Clustering Algorithm for 3-D MR Image Segmentation," IEEE Trans. on Medical Imaging, Vol.22, No.9, pp.1063-1075, 2003.

[ 9 ] M. E. Algorri and F. Flores-Mangas, "Classification of Anatomical Structures in MR Brain Images Using Fuzzy Parameters," IEEE Trans. on Biomedical Engineering, Vol.51, No.9, pp.1599-1608, 2004.

[10] S. Chen and D. Zhang, "Robust Image Segmentation Using FCM with Spatial Constraints Based on New Kernel-Induced Distance Measure," IEEE Trans. on Syst., Man, Cybern. B, Vol.34, No.4, pp.1907-1916, 2004.

[11] J. Luo, Y. Zhu, P. Clarysse and I. Magnin, "Correction of Bias Field in MR Images Using Singularity Function Analysis," IEEE Trans. on Medical Imaging, Vol.24, No.8, pp.1067-1085, 2005.

[12] A. Madabhushi and J. K. Udupa, "Interplay between intensity standardization and inhomogeneity correction in MR image processing," IEEE Trans. on Medical Imaging, Vol.24, No.5, pp.561- 576, 2005.

[13] A. Simmons, P. S. Tofts, G. J. Barker and S. R. Arridge, "Sources of Intensity Nonuniformity in spin echo images at $1.5 \mathrm{~T}, "$ Magnetic Resonance in Medicine, Vol.32, No.1, pp.121-128, 1994.

[14] Y. Sato, C. Westin, A. Bhalerao, S. Nakajima, N. Shiraga, S. Tamura and R. Kilinis, "Tissue Classification Based on 3D Local Intensity Structure for Vol- ume Rendering," IEEE Trans. on Visualization and Comp. Graphics, Vol.6, No.2, pp.160-180, 2000.

[15] S. Luo, W. Li, N. Li and J. Liu, "Evaluation of Brain Tissue Classification in MR Images Using Simulated Brain Database," Proc. of the 22nd Annual EMBS Int. Conf., pp.678-681, 2000.

[16] http://www.fmrib.ox.ac.uk/fsl/ FMRIB Software Library

[17] Y. Hata, S. Kobashi, S. Hirano, H. Kitagaki and E. Mori, "Automated Segmentation of Human Brain MR Images Aided by Fuzzy Information Granulation and Fuzzy Inference," IEEE Trans. on Syst., Man, Cybern. C, Vol.30, No.3, pp.381-395, 2000.

[18] S. Kobashi, N. Kamiura and Y. Hata, "Fuzzy information granulation on segmentation of human brain MR images," J. Japan Society for Fuzzy Theory and Systems, Vol.10, No.1, pp.117-125, 1998.

(2007年 6 月 14 日 受付)

(2007年11月24日 採録)

[Contact Address ]

Graduate School of Engineering, University of Hyogo

2167, Himeji, Shosha, Hyogo 671-2280, JAPAN

Syoji KOBASHI

TEL : + 81-79-267-4989

FAX : + 81-79-267-4989

E-mail : kobashi@eng.u-hyogo.ac.jp 


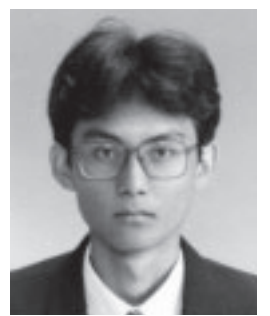

\section{Syoji KOBASHI [member]}

received the B.E. (1995), M.E. (1997) and D.E. (2000) from the Himeji Institute of Technology (Hyogo, Japan). He is currently an associate professor with the University of Hyogo. His research interests include soft computing approach to medical signal/image processing and human brain functions. He received the Joseph F. Engelberger Best Paper Award at the 2nd World Automation Congress in 2000, the Highlighted Technical Paper Award at the Australian and New Zealand Intelligent Information Systems in 2003, and the IEEE EMBS Japan Young Investigators Competition from IEEE EMBS Japan Chapter in 2003. He is a member of the IEEE.

\section{Noriko INOUE [non-member]}

received B. Arts in Literature from Kobe City University of Foreign Studies, Hyogo, Japan in 1980. She is currently a Research Coordinator for Industries \& University Collaboration Programs, Medical Center for Translational Research, Osaka University Hospital. She was a Research Coordinator in Brain Function Research Institute from September 1999 to March 2004. Her research interests include imaging of higher brain function and medico-engineering techniques such as molecular imaging and nanobiology, as well as regenerative medicine.

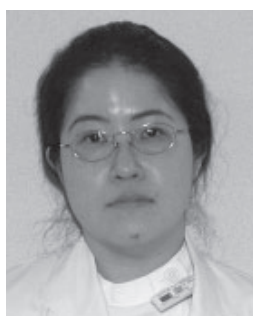

\section{Mieko MATSUI [non-member]}

became MD in 1992 after graduation from Kobe University School of Medicine, Hyogo, Japan, and obtained PhD from Kobe University in 1998. She is currently a physician in Department of Radiology, Kakogawa Municipal Hospital. She has plodded along in this work to date after being affiliated with the BF Research Institute, Osaka, Japan as a senior researcher in 2002. Her research interest is mainly related to the diagnostic and therapeutic imaging of dementia and its related disorders. She is a member of International Society for Magnetic Resonance in Medicine, Japan Radiological Society, Japanese Society for Magnetic Resonance in Medicine, Japanese Society of Nuclear Medicine, Japanese Society of Cognitive Neuroscience and Japanese Society of Angiography and Interventional Radiology.

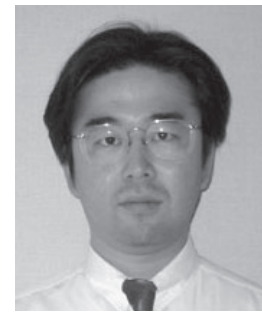

\section{Katsuya KONDO [non-member]}

received the B.E., M.E., and D.E. degrees from Keio University, Yokohama, Japan, in 1989, 1991 and 1997, respectively. He is an associate professor with University of Hyogo. His research interests include signal processing and computer vision. Dr. Kondo received World Automation Congress Albertos Best Paper Award in 2004. He is a member of the Institute of Electronics, Information and Communication Engineers and IEEE. 


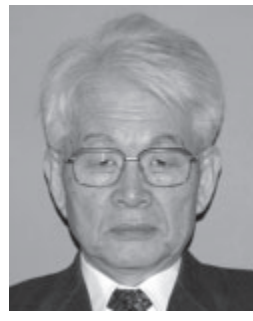

\section{Tohru SAWADA [non-member]}

became an MD in 1961 after graduation from School of Medicine Keio University, Tokyo, Japan, and obtained $\mathrm{PhD}$ from Keio University in 1970. He is currently the director of Department of Rehabilitation, Aino Hospital, Osaka, Japan and a visiting professor of neurology both of School of Medicine Keio University, Tokyo, Japan and Suchou Medical College in Suchou, China. He was nominated as the Executive Director of BF Research Institute in 1997 and took charge of whole research works performed in the institute from 1997 to 2004. Dr. Sawada published various medical papers related to cerebrovascular diseases, cerebral circulation, higher cortical functions, or dementia, and he experienced editorial board members of international journals such as "Cerebrovascular Diseases", "Alzheimer's Disease and Related Disorders", or "Japanese Stroke Journal". He is presently a member of the steering committee of the Japanese Stroke Society, and emeritus member of Japanese Society of Cerebral Circulation and Metabolism. He is also one of council members of the Japanese Society of Neurology and Stroke Division of American Heart Association.

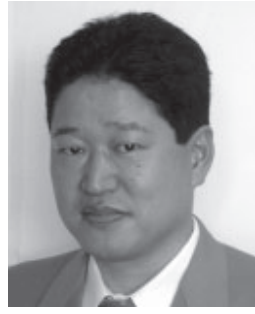

Yutaka HATA [member]

received the B.E.(1984), M.E.(1986), and D.E. (1989) degrees from Himeji Institute of Technology, Japan. He is currently a Professor with University of Hyogo. His research interests include fuzzy logic, medical imaging, ultrasound systems. He received World Automation Congress Contribution Award (2002, 2004, 2006) Joseph F. Engelberger Best Paper Award and Best Paper Award at 2000 World Automation Congress and a Distinctive Contributed Paper Award at 1999 IEEE Int. Sympo. on Multiple-Valued Logic. He is a senior member of the IEEE. 